\title{
ON A CHARACTERIZATION OF THE EXPONENTIAL FUNCTION AND THE COSINE FUNCTION BY FACTORIZATION II
}

\author{
By Mitsuru Ozawa
}

1. In our previous paper [1] we proved the following

THEOREM A. Let $F(z)$ be an entire function for which there exist polynomials $P_{m}(z)$ of degree $m$ and entrre functions $f_{m}(z)$ so that $F(z)=P_{m}\left(f_{m}(z)\right)$ for $m=2^{j}$, $j=1,2, \cdots$ and for $m=3$. Then $F(z)$ is either $A e^{H(z)}+B$ or $A \cos \sqrt{H(z)}+B$ with constants $A, B$ and an entire function $H(z)$.

In this paper we shall give an application of this theorem.

THEOREM 1. Let $F(z)$ be an entrre function for which

$$
F(z)=P_{2}\left(F\left(\frac{z}{n}\right)\right)=P_{3}\left(F\left(\frac{z}{m}\right)\right)
$$

with polynomials $P_{k}$ of degree $k$ and positive integers $n, m$. Then $F(z)$ is either $A e^{a z}+B$ or $A \cos a z+B$ or $A \cos \sqrt{a z}+B$ with constants $A, B$ and $a$.

This theorem gives again a characterization of exp and cos. It seems to the present author that there is another proof depending on the power series expansion. If we omit the condition $F(z)=P_{3}\left(F\left(\frac{z}{m}\right)\right)$ in our theorem, we cannot say that theorem 1 holds.

In this theorem we may put $m, n$ as non-zero constants and we have the same conclusion.

2. Proof of Theorem 1. Evidently

$$
F(z)=P_{2^{j}}\left(F\left(\frac{z}{n^{\jmath}}\right)\right)=P_{3}\left(F\left(\frac{z}{m}\right)\right)
$$

for $\jmath=1,2,3, \cdots$. Hence Theorem A implies that $F(z)$ is either $A e^{H(z)}+B$ or $A \cos \sqrt{H(z)}+B$. By $F(z)=P_{2}(F(z / n))$ we have further $m(r, F) \sim 2 m(r / n, F)$ as $r \rightarrow \infty$. For $r \geqq r_{0}$

Received June 9, 1977 


$$
(1-\varepsilon) 2 m\left(\frac{r}{n}, F\right) \leqq m(r, F) \leqq(1+\varepsilon) 2 m\left(\frac{r}{n}, F\right) .
$$

For $r_{0} \leqq r_{2} \leqq n r_{0}=r_{1}$ and $r=n^{p} r_{2}$

$$
\varlimsup_{p \rightarrow \infty} \frac{\log m\left(n^{p} r_{2}, F\right)}{\log n^{p} r_{2}} \leqq \frac{\log 2+\log (1+\varepsilon)}{\log n}
$$

and

$$
\lim _{p \rightarrow \infty} \frac{\log m\left(n^{p} r_{2}, F\right)}{\log n^{p} r_{2}} \geqq \frac{\log 2+\log (1-\varepsilon)}{\log n} .
$$

Hence the order of $F$ is equal to $\log 2 / \log n$. Evidently $n \geqq 2$. If $F(z)=A e^{H(z)}+B$, its order $\geqq 1$ and so $n$ should be equal to 2 . Therefore $F(z)=A_{1} e^{a z}+B$. If $F(z)$ $=A \cos \sqrt{H(z)}+B$, its order should be a half integer and hence $H(z)$ is either $a z+a_{0}$ if $n=4$ or $a z^{2}+a_{1} z+a_{0}$ if $n=2$. Let $P_{2}(w)$ be $C_{2} w^{2}+C_{1} w+C_{0}$. Then by $F(z)=P_{2}(F(z / 2))$ in the latter case

$$
\begin{aligned}
A \cos & \sqrt{a z^{2}+a_{1} z+a_{0}}+B \\
= & \frac{C_{2} A^{2}}{2} \cos \sqrt{a z^{2}+2 a_{1} z+4 a_{0}}+\left(2 C_{2} A B+C_{1} A\right) \cos \sqrt{\frac{a}{4} z^{2}+\frac{a_{1}}{2} z+a_{0}} \\
& +C_{2} B^{2}+C_{1} B+C_{0}+\frac{C_{2}}{2} A^{2} .
\end{aligned}
$$

Without appealing to the impossibility of Borel's identity we can proceed in the following manner. The left hand side term is symmetric with respect to $-a_{1} / 2 a$ and the right hand side term is symmetric with respect to $-a_{1} / a$. Hence $a_{1}=0$. Next consider the asymptotic behavior of both sides for $\sqrt{a z^{2}}=i y$ with real $y$ for $y \rightarrow \infty$. Then $2 A=C_{2} A^{2}, 2 C_{2} A B+C_{1} A=0$. Hence

$$
\begin{aligned}
& A \cos \sqrt{a z^{2}+a_{0}}+B \\
& \quad=A \cos \sqrt{a z^{2}+4 a_{0}}+C_{2} B^{2}+C_{1} B+C_{0}+\frac{C_{2}}{2} A^{2} .
\end{aligned}
$$

Let us put $a z^{2}+a_{0}=\left(2 n \pi+\frac{\pi}{2}\right)^{2}$. Then

$$
B=A \sin \frac{3 a_{0}}{2 n \pi+\frac{\pi}{2}}\left(\frac{1}{2}+O\left(\frac{1}{n^{2}}\right)\right)+C_{2} B^{2}+C_{1} B+C_{0}+\frac{C_{2}}{2} A^{2} .
$$

Hence $a_{0}=0$ and $B=C_{2} B^{2}+C_{1} B+C_{0}+C_{2} A^{2} / 2$. Therefore

$$
F(z)=A \cos \sqrt{a} z+B .
$$

In the former case 


$$
\begin{aligned}
A \cos \sqrt{a z+a_{0}}+B \\
=C_{2} \frac{A^{2}}{2} \cos \sqrt{a z+4 a_{0}}+\left(2 A B C_{2}+C_{1} A\right) \cos \sqrt{\frac{a}{4} z+a_{0}} \\
\quad+\frac{C_{2}}{2} A^{2}+C_{2} B^{2}+C_{1} B+C_{0} .
\end{aligned}
$$

Quite similarly we have

$$
\begin{gathered}
a_{0}=0, \quad A=\frac{C_{2}}{2}-A^{2}, \quad 2 A B C_{2}+C_{1} A=0, \\
B=\frac{C_{2}}{2} A^{2}+C_{2} B^{2}+C_{1} B+C_{0} .
\end{gathered}
$$

Hence

$$
F(z)=A \cos \sqrt{a z}+B .
$$

This completes the proof of our theorem.

\section{BIBLIOGRAPHY}

[1] OzAwa, M., On a characterization of the exponential function and the cosine function by factorization. Kodai Math. J. 1 (1978), 45-74.

Department of Mathematics

TOKYO INSTITUTE OF TECHNOLOGY

Oh-okayama, Meguro-ku, Tokyo, Japan 